\section{FRI0442 1 APPROPRIATE USE OF SEROLOGY TESTS FOR THE DIAGNOSIS OF LYME DISEASE. EXPERIENCE IN AN URBAN AREA}

D. Lobo Prat ${ }^{1}$, L. Sainz Comas ${ }^{1}$, V. Pomar ${ }^{2}$, A. M. Millán Arciniegas ${ }^{1}$, H. Park ${ }^{1}$ A. García-Guillén ${ }^{1}$, S. Jeria ${ }^{1}$, A. Laiz ${ }^{1}$, B. Magallares ${ }^{1}$, I. Castellví ${ }^{1}$, P. Moya ${ }^{1}$, C. Díaz-Torné ${ }^{1}$, S. P. Fernandez-Sanchez ${ }^{1}$, H. Corominas ${ }^{1} .{ }^{1}$ Hospital de la Santa Creu i Sant Pau, Barcelona, Spain; ${ }^{2}$ Hospital de la Santa Creu i Sant Pau, Infectious Diseases, Barcelona, Spain

Background: Lyme disease (LD) is a multisystemic animal-borne disease caused by spirochetes of the Borrelia burgdorferi s.l complex and transmitted by ticks of the species /xodes ricinus. In Spain, most cases occur in rural areas of the north-east region with a peak of maximum incidence between spring and early autumn. The diagnosis is based on a history of potential exposure to ticks, the recognition of characteristic clinical manifestations and serological testing.

Objectives: To assess the suitability of serological study for the diagnosis of LD in an urban area.

Methods: Retrospective observational study that included all LD serology tests made between April 2017 and September 2019 at a tertiary hospital in Barcelona covering a population of 450,000 people. Demographic data and the medical department that requested the serology test were collected along with serology test results. The medical records of patients with positive serology were consulted to identify which patients were finally diagnosed with LD along with their clinical manifestations, treatment and outcome.

Results: A total of 574 serological tests were included and 78 (13.59\%) of them were positive. Only $1.04 \%$ (6) of all serological tests belonged to patients finally diagnosed with LD. The department that made most requests was Neurology (37.3\%) followed by Infectious Diseases (21\%), Internal Medicine (14.5\%), Emergency Medicine (4.7\%), Dermatology (4.5\%), Critical Care Medicine $(2.3 \%)$ and Rheumatology $(2.1 \%) .50 \%$ of the diagnosed patients were women with a mean age of $57.7 \pm 7.7 \mathrm{DE}$ years. In $50 \%$ of diagnosed cases, patients remembered a tick bite during activities in the mountain or rural areas. The most common clinical manifestations were erythema migrans $(67 \%)$, non-inflammatory arthralgias $(50 \%)$, fatigue and malaise (67\%), together with one case of meningoencephalitis and one of knee monoarthritis. All diagnosed patients received antibiotic treatment with ceftriaxone $(33 \%)$ or doxycycline $(66 \%)$. Only one patient presented post-Lyme syndrome.

The serological test for LD in our center had a total individual cost of 15.75 eur, so the cost of the 574 requests was $9,040.5$ eur. 7,812 eur corresponded to negative results and 1,134 eur to false positive results.

Conclusion: Our study indicates the overuse of diagnostic testing for LD with implications for patient care and cost-effective health management. In the absence of a history of potential exposure to infected vector ticks or characteristic clinical manifestations, unnecessary microbiological tests should not be performed.

Disclosure of Interests: David Lobo Prat: None declared, Luís Sainz Comas: None declared, Virginia Pomar: None declared, Ana Milena Millán Arciniegas: None declared, HyeSang Park: None declared, Andrea García-Guillén: None declared, Sicylle Jeria: None declared, Ana Laiz: None declared, Berta Magallares: None declared, Ivan Castellví Consultant of: Boehringer Ingelheim, Actelion, Kern Pharma, Speakers bureau: Boehringer Ingelheim, Actelion, Bristol-Myers Squibb, Roche, Patricia Moya: None declared, Cesar Díaz-Torné: None declared, Susana P. Fernandez-Sanchez: None declared, Hector Corominas: None declared

DOI: 10.1136/annrheumdis-2020-eular.6586

\section{FRI0443 \\ CLINICAL CHARACTERISTICS AND RELATED FACTORS OF COMMON RHEUMATIC DISEASES COMPLICATED WITH TUBERCULOSIS INFECTION}

L. Long ${ }^{1}$, G. Tang ${ }^{1}$, Y. Han ${ }^{1}$, Q. Peng ${ }^{1}$, J. Liu', X. Chen ${ }^{1}$, Q. Zhou ${ }^{1} .{ }^{1}$ Sichuan Provincial People's Hospital, School of Medicine, University of Electronic Science and Technology of China, Chengdu, China

Background: Rheumatoid arthritis (RA), systemic lupus erythematosus (SLE), and syndrome(SS) are common rheumatic diseases with high incidence. Patients with those rheumatic diseases are at high risk of tuberculosis (TB) infection. However, manifestations can be atypical and easily confused with those of rheumatic disease itself. For those patients, diagnosis is usually much more difficult and further make treatment delayed. Sometimes it may lead to mistreatment. Therefore, it is important to recognize the clinical characteristics of those patients.

Objectives: To explore the clinical characteristics and high risk factors of common systemic rheumatism complicated with tuberculosis infection.

Methods: A total of 3,906 cases of RA, SLE, and SS common systemic rheumatism diagnosed in the People's Hospital of Sichuan Province from
January 2007 to January 2017 were collected with carefully exclusion with other infectious diseases and neoplastic disease. One hundred and five patients with TB were included as infection group, including 42 cases of RA, 41 cases of SLE, and 22 cases of SS. In the control group, 84 patients with RA, 82 patients with SLE, and 44 patients with SS were randomly selected from the corresponding rheumatoid non-infected patients hospitalized during the same period.

Results: Fever was the most common symptom among 42 cases of RA, 41 cases of SLE, and 22 cases of SS with TB, accounting for $83.3 \%, 92.7 \%$, and $68.2 \%$, respectively. Cough, weight loss or fatigue was less common. For 41 cases of SLE and 22 cases of SS with TB, the proportion of pulmonary was $46.3 \%, 59.01 \%$, respectively. In TB infection group, 27 cases of RA, 21 cases of SLE, and 13 cases of SS with TB had two or more chest CT findings, accounting for $59 \%, 57 \%, 62 \%$, respectively. Lesions located in the posterior or posterior segment which TB usually affected were 9 cases(33.3\%),9cases $(42.9 \%), 6$ cases $(27.2 \%)$, respectively. The daily average dose of hormones within 1 year in TB infection group was higher than that in the control group $(P<0.05)$. For SLE patients, lower counts of CD4+TL were found in TB infection group $(P<0.05)$, while no such differences were found in RA and SS group.

Conclusion: Patients with RA who have TB infection are mainly pulmonary TB For SLE and SS patients, the chance of pulmonary tuberculosis and extra-pulmonary tuberculosis is similar.Symptoms of RA, SLE, SS with TB, such as fever, cough, weight loss, fatigue, are similar with the primary disease or other infection. Chest imaging is diversity. It is difficult to diagnose.Daily average dose of hormone within one year may be a common risk factor for RA, SLE and SS patients with TB. Decreased CD4+TL may also be a risk factor for SLE patients with TB.

\section{eferences:}

[1] Cantini F, Nannini C, Niccoli L, et al. Risk of Tuberculosis Reactivation in Patients with Rheumatoid Arthritis, Ankylosing Spondylitis, and Psoriatic Arthritis Receiving Non-Anti-TNF-Targeted Biologics[J]. Mediators of Inflammation, 2017, 2017(6):1-15

[2] Ruangnapa K, Dissaneewate P, Vachvanichsanong P. Tuberculosis in SLE patients: rare diagnosis, risky treatment.[J]. Clinical \& Experimental Medicine, 2015, 15(3):429-432.

[3] Manuela D F, Bruno L, Martina S, et al. Lung Infections in Systemic Rheumatic Disease: Focus on Opportunistic Infections[J]. International Journal of Molecular Sciences, 2017, 18(2):293-315.

[4] Disseminated tuberculosis masquerading as a presentation of systemic lupus erythematosus.Li JC, Fong W, Wijaya L, Leung YY.Int J Rheum Dis. 2017 Oct 2. doi: 10.1111/1756-185X.13195.

[5] Handa R, Upadhyaya S, Kapoor S, et al. Tuberculosis and biologics in rheumatology: India - A special situation[J]. International Journal of Rheumatic Diseases, 2017, 51(2):115.

Disclosure of Interests: None declared

DOI: 10.1136/annrheumdis-2020-eular.3717

\section{FRI0444 HPVB19 INFECTION IN ADULT 53 CASES}

M. Minoda ${ }^{1}$, M. Suda ${ }^{1}$, K. Muranaka ${ }^{1} .{ }^{1}$ Suwa Central Hospital, Rheumatology, Chino city, Japan

Background: Human parvovirus B19(HPVB19) is a member of the large Parvoviridae family and, as a human pathogen, is responsible for various diseases. 1) In children, HPVB19 infections usually presents as red rash, which is often described as a"slapped cheek." However, the presentation varies in adults. ${ }^{2}$ HPVB19 outbreaks mostly occur in the winter and spring. ${ }^{1)}$ The serum IgM test is the preferred means for diagnosing acute HPVB19 infections. ${ }^{2)}$

Objectives: We investigated the epidemiology, clinical symptoms, and time course of HPVB19 infections in real-world practice by examining patients treated at a community hospital in Japan.

Methods: We studied adult patients with HPVB19 infections at Suwa Central Hosptial from April 2010 to November2019. Patients who were age 18 years or older, anti HPV B19 IgM antibody-positive, and symptomatic were included. We retrospectively examined the patients' medical records to determine onset days, clinical symptoms and antinuclear antibody(ANA)-postitive rates. We defiend day 1 as the day when clinical symptoms first appeared.

Results: 53 patients were included in the study. Patient characteritistics were mean age 43 years, female $79.2 \%$, mean period from onset to diagnosis 13days. Epidemic season were 4year cycle. (Figure 1) Clinical symptoms and time course from onset day were upper respiratory symptoms(17.0\%, 1.7 days) fever( $54.7 \%, 1.9$ days $)$, abdominal symptoms $(15.1 \%, 4.4$ days $)$, edema( $47.2 \%$, 5.6 days $)$, arthralgia(71.7\%, 6.6days), itching $(15.1 \%, 6.9$ days $)$, rash $(62.3 \%$, 8.9days) and lymphoadenopathy(30.2\%, 10.2days). (Figure 2) ANA positive rate was $50 \%(13 / 26)$ and average was 92.3 . 